\title{
INTEGRAL/IBIS observations of a hard X-ray outburst in high-mass X-ray binary 4U 2206+54 (Research Note)
}

\begin{abstract}
W. Wang
National Astronomical Observatories, Chinese Academy of Sciences, Beijing 100012, PR China

e-mail: wangwei@bao.ac.cn

Received 5 May 2010 / Accepted 20 July 2010

ABSTRACT

Aims. 4U 2206+54 is a wind-fed high-mass X-ray binary with a main-sequence donor star. The nature of its compact object has been recently identified as a slow-pulsation magnetized neutron star.

Methods. INTEGRAL/IBIS observations have a long-term hard X-ray monitoring of 4U 2206+54 and detected a hard X-ray outburst around 15 December 2005 combined with the RXTE/ASM data.

Results. The hard X-ray outburst had a double-flare feature with a duration of $\sim 2$ days. The first flare showed a fast rise and long-term decaying light curve about $15 \mathrm{~h}$ with a peak luminosity of $\sim 4 \times 10^{36} \mathrm{erg} \mathrm{s}^{-1}$ from $1.5-12 \mathrm{keV}$ and a hard spectrum (only significantly seen above $5 \mathrm{keV}$ ). The second one had the mean hard X-ray luminosity of $1.3 \times 10^{36} \mathrm{erg} \mathrm{s}^{-1}$ from $20-150 \mathrm{keV}$ with a modulation period at $\sim 5550 \mathrm{~s}$ which is the pulse period of the neutron star in $4 \mathrm{U} 2206+54$. Its hard X-ray spectrum from $20-300 \mathrm{keV}$ can be fitted by a broken power-law model with the photon indexes $\Gamma_{1} \sim 2.3$, and $\Gamma_{2} \sim 3.3$, and the break energy is $E_{b} \sim 31 \mathrm{keV}$ or by a bremsstrahlung model of $k T \sim 23 \mathrm{keV}$.

Conclusions. We suggest that the hard X-ray flare could be induced by suddenly enhanced accreting dense materials from stellar winds hitting the polar cap region of the neutron star. This hard X-ray outburst may be a link to supergiant fast X-ray transients though 4U 2206+54 has a different type of companion.
\end{abstract}

Key words. stars: individual: 4U 2206+54 - stars: neutron - X-rays: binaries - X-rays: bursts

\section{Introduction}

The massive X-ray binary 4U 2206+54 was discovered by the Uhuru satellite (Giacconi et al. 1972). The optical counterpart was identified as an $09.5 \mathrm{~V}$ star with a high $\mathrm{He}$ abundance and has a distance of $\sim 2.6 \mathrm{kpc}$ (Blay et al. 2006). Without a circumstellar disk around the donor, the material for accretion and production of high-energy emission must come from stellar wind (Negueruela \& Reig 2001). The wind's terminal velocity of 4U2206+54 has a low value of $\sim 350 \mathrm{~km} \mathrm{~s}^{-1}$ (Ribo et al. 2006), so assuming an eccentric orbit and using the BondiHoyle formalism wind-fed accretion could produce X-ray luminosity and variability $\left(L_{x} \sim 10^{33}-10^{35} \mathrm{erg} \mathrm{s}^{-1}\right)$ as seen by RXTE, BepposSAX, Swift and INTEGRAL (Torrejon et al. 2004; Masetti et al. 2004; Blay et al. 2005; Corbet et al. 2007; Wang 2009).

X-ray monitoring of $4 \mathrm{U} 2206+54$ by RXTE has suggested a modulation period of 9.6 days (Corbet \& Peele 2001) which may be an orbit period. Recent SWIFT/BAT observations (Corbet et al. 2007) and RXTE/ASM data (Wang 2009) found a modulation of $\sim 19.12$ days consistent with twice the 9.6-day period.

The nature of the compact object in $4 \mathrm{U} 2206+54$ has been disputed for a long time (Negueruela \& Reig 2001; Corbet $\&$ Peele 2001). Recent reports of detecting cyclotron resonant absorption lines at $\sim 30 \mathrm{keV}$ and $60 \mathrm{keV}$ suggested a magnetized neutron star with a magnetic field of $\sim 3.3 \times 10^{12} \mathrm{G}$ by INTEGRAL observations (Wang 2009; Blay et al. 2005). The reports of possible $5500 \mathrm{~s}$ pulsations in light curves of 4U 2206+54 from RXTE, INTEGRAL and Suzaku observations suggested that it would be a X-ray pulsar (Reig et al. 2009; Wang 2009; Finger et al. 2010).

In this work, we report a long-duration hard X-ray flare in 4U 2206+54 discovered by INTEGRAL/IBIS and RXTE/ASM observations. We first introduce the observations of INTEGRAL/IBIS and RXTE/ASM telescopes in Sect. 2. The main features of the hard X-ray flare in 4U 2206+54 are described in Sect. 3. The summary and discussions of the possible origins of this hard X-ray flare are delineated in Sect. 4.

\section{Observations}

\subsection{INTEGRAL/IBIS}

The hard X-ray source 4U 2206+54 was observed during the INTEGRAL pointed observations of the Cassiopeia region around Dec. 2005. The hard X-ray flare were captured by the low-energy detector (called ISGRI) of the Imager (IBIS, Lebrun et al. 2003) aboard INTEGRAL. The IBIS-ISGRI scientific data analysis was carried out using the Off-line Scientific Analysis (OSA) software version 7.0 (Goldwurn et al. 2003) provided by the INTEGRAL Science Data Center (ISDC). Individual pointings in each satellite revolution (3 days) processed with OSA 7.0 were mosaicked to create sky images for source detections. We used the 20-40 keV band for source detection and to quote fluxes (Table 1). For Rev 387, 4U 2206+54 has a detection significance level of $\sim 45 \sigma$ with an average IBIS/ISGRI count rate up to $\sim 11.9 \mathrm{cts} \mathrm{s}^{-1}$ in the energy band of $20-40 \mathrm{keV}$. $4 \mathrm{U} 2206+54$ 
appeared in the outburst state around Dec. 15, 2005 in the hard $\mathrm{X}$-ray bands.

We derived the hard X-ray light curve of 4U 2206+54 in the band of 20-40 keV from 2005 Dec. 11 to Dec. 19 (see Fig. 1). 4U 2206+54 was unfortunately outside the field of view (FOV) of IBIS sometimes, and the data were also screened for solar-flare events and erratic count fluctuations due to passages through the Earth's radiation belts, so several data gaps appeared in the light curve of $4 \mathrm{U} 2206+54$ (Fig. 1). From the available IBIS data, a strong hard X-ray outburst was still detected around Dec. 15-16, 2005, which lasted $\sim 10^{5} \mathrm{~s}$.

\subsection{RXTE/ASM}

RXTE had no pointed observations on the source 4U 2206+54 during December 2005. Fortunately, the source was regularly monitored by the All Sky Monitor (ASM) onboard RXTE. The ASM consists of three Scanning Shadow Cameras (SSCs) mounted on a rotating drive assembly. Each camera has a field of view of $6^{\circ} \times 90^{\circ}$. The assembly "dwells" at a fixed position for $\sim 90 \mathrm{~s}$, followed by a rotation of $6^{\circ}$. Each camera has a position sensitive proportional counter, and the data is analyzed to give not only the total source intensity in the $1.5-12 \mathrm{keV}$ band, but also the intensity in each of 3 energy bands: $1.5-3 \mathrm{keV}, 3-5 \mathrm{keV}$, and 5-12 keV. The ASM data are available in two forms: count rates from individual 90-s dwells and a one-day average for each source.

From the archival dataset ${ }^{1}$ provided by the surveys of the All-Sky Monitor (ASM) aboard RXTE, we obtained the dwellby-dwell light curve (1.5-12 keV) of 4U 2206+54 during the hard X-ray outburst (Fig. 1). ASM data filled up the data gap before the hard X-ray burst obtained from the IBIS data. Therefore, the complete observed features of the super-long duration hard X-ray outburst in 4 U $2206+54$ were derived by using both ASM and IBIS observations.

\section{Results}

The hard X-ray outburst occurred at UT 2005 Dec. 15.1085 and lasted over 2 days. This peculiar flare showed a double-flare feature in the light curve. We present the properties of the first and second flares separately in the following.

\subsection{The first flare}

The first flare was only captured by the ASM data and had a peak flux of $\sim 100 \mathrm{mCrab}$, and it decayed to $\sim 20 \mathrm{mCrab}$. It appeared to rise sharply to a peak, and then the flux decayed over a long time period of $\sim 15 \mathrm{~h}$. The peak flux in the energy range of $1.5-12 \mathrm{keV}$ is $(3.3 \pm 0.9) \times 10^{-9} \mathrm{erg} \mathrm{cm}^{-2} \mathrm{~s}^{-1}$, and there is a total fluence of $\sim 5.3 \times 10^{-5} \mathrm{erg} \mathrm{cm}^{-2}$. For a distance of $2.6 \mathrm{kpc}$ (Blay et al. 2006), the peak luminosity at the first flare reached $\sim 3.6 \times 10^{36} \mathrm{erg} \mathrm{s}^{-1}$, and total energy release during the first flare is $\sim 6 \times 10^{40} \mathrm{erg}$ in the energy range of $1.5-12 \mathrm{keV}$. This X-ray flare cannot be significantly detected below $5 \mathrm{keV}$ (Fig. 2), implying a hard spectrum in the first flare.

\subsection{The second flare}

The second flare appeared about $\sim 15 \mathrm{~h}$ after the first one. It was observed by both ASM and IBIS. ASM only obtained a few data

1 http://xte.mit.edu/asmlc/ASM.html

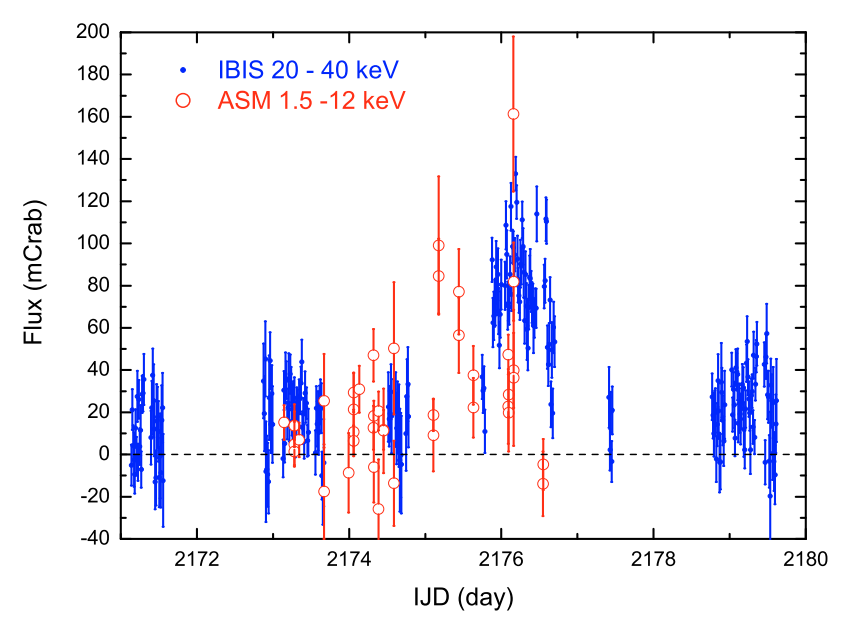

Fig. 1. Hard X-ray lightcurves of 4 U 2206+54 from IJD 2171 to 2180 (IJD = MJD-51544) in the energy ranges of $1.5-12 \mathrm{keV}$ from the RXTE/ASM data and 20-40 keV observed by INTEGRAL/IBIS. The flux unit of 1 Crab corresponds to $2.2 \times 10^{-8} \mathrm{erg} \mathrm{cm}^{-2} \mathrm{~s}^{-1}$ in the band of $1.5-12 \mathrm{keV}$ and $7.6 \times 10^{-9} \mathrm{erg} \mathrm{cm}^{-2} \mathrm{~s}^{-1}$ in the $20-40 \mathrm{keV}$ band.

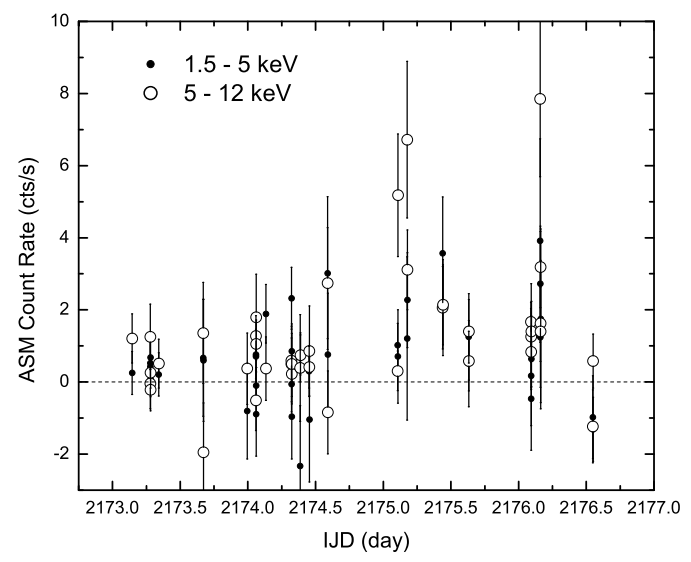

Fig. 2. The ASM lightcurves of the X-ray binary 4U 2206+54 in two energy bands: $1.5-5 \mathrm{keV}$, and $5-12 \mathrm{keV}$. The X-ray flare cannot be significantly detected below $5 \mathrm{keV}$.

points for the second flare, but IBIS showed the detailed variability information (Fig. 3). In the energy range of 20-40 keV, the second flare lasted more than $8 \times 10^{4} \mathrm{~s}$. Multiple mini-peak features appeared in the second flare. The power spectrum analysis showed a significant modulation period at $\sim 5550 \pm 50 \mathrm{~s}$ (Fig. 3), which confirms the pulsation period of the neutron star in 4U 2206+54 (Reig et al. 2009; Wang 2009). The folded light curve is shown in Fig. 4. The pulse fraction defined as the ratio of the pulse maximum minus the minimum to the maximum is estimated as $\sim 30 \%$ during the flare. Reig et al. (2009) find a pulse fraction of $\sim 50 \%$ with RXTE and INTEGRAL data, and Wang (2009) obtained a pulse fraction of $\sim 80 \%$. The pulse fraction during the outburst appears to be lower than those in the other states.

We obtained the spectrum for the second flare from 20-300 keV from IBIS observations (see Fig. 5). This spectrum can be fitted well by two different models: a thermal bremsstrahlung model with $k T \sim 23.4 \pm 1.3 \mathrm{keV}$ (reduced 

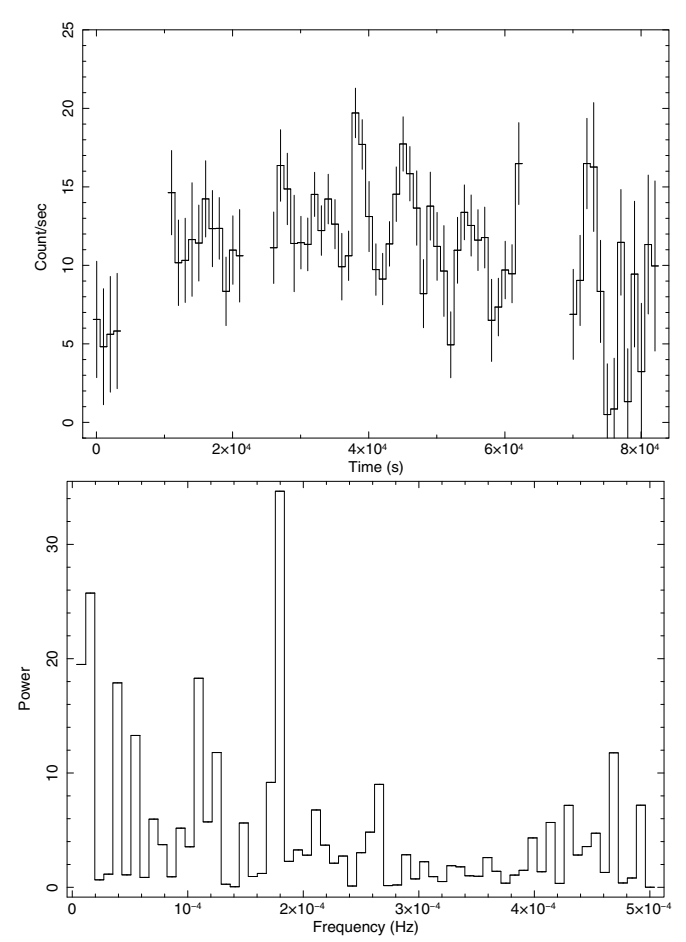

Fig. 3. Top: the light curve of the second flare of the hard X-ray outburst by INTEGRAL/IBIS. Bottom: the power spectrum of the INTEGRAL/IBIS light curve of 4U 2206+54 during the second flare. A significant period at $5550 \pm 50 \mathrm{~s}$ is detected in the light curve.

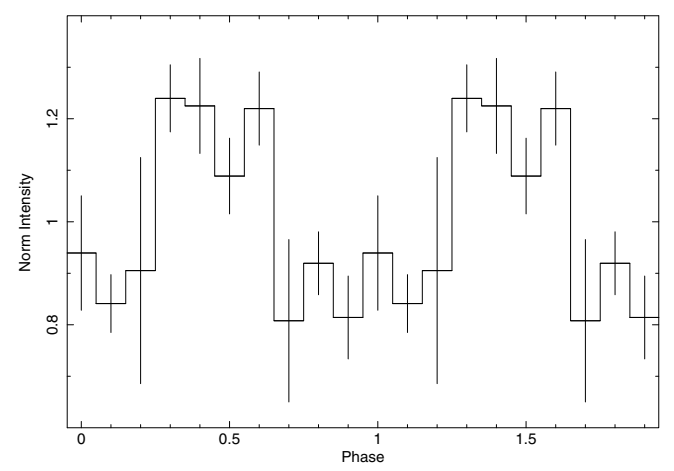

Fig. 4. The IBIS/ISGRI background-subtracted light curve (20-40 keV) of $4 \mathrm{U} 2206+54$ during the second flare folded at a pulsation period (5550 s). The pulse profile is repeated once for clarity.

$\chi^{2} \sim 0.81,9$ d.o.f.); a broken power-law model with the photon indexes $\Gamma_{1} \sim 2.3 \pm 0.2, \Gamma_{2} \sim 3.3 \pm 0.3$, and the break energy is $E_{b} \sim 31.2 \pm 1.7 \mathrm{keV}$ (reduced $\chi^{2} \sim 0.77,7$ d.o.f.). No cyclotron absorption lines are found in the spectrum of the different fits.

The average flux during the second flare from $20-150 \mathrm{keV}$ energy band is $\sim(1.2 \pm 0.1) \times 10^{-9} \mathrm{erg} \mathrm{cm}^{-2} \mathrm{~s}^{-1}$, corresponding to a mean luminosity of $1.3 \times 10^{36} \mathrm{erg} \mathrm{s}^{-1}$ in the range of $20-150 \mathrm{keV}$ assuming a distance of $2.6 \mathrm{kpc}$. Then the total released energy of the second flare with the duration of $\sim 10^{5} \mathrm{~s}$ is about $10^{41} \mathrm{erg}$.

\section{Summary and discussion}

In this paper we have reported the INTEGRAL/IBIS and RXTE/ASM observations of a 2-day long hard X-ray outburst in 4U 2206+54 that harbors a highly magnetized neutron star (Wang 2009). This outburst is peculiar in two ways. (1) A double-flare feature, the second flare occurred about $15 \mathrm{~h}$ after

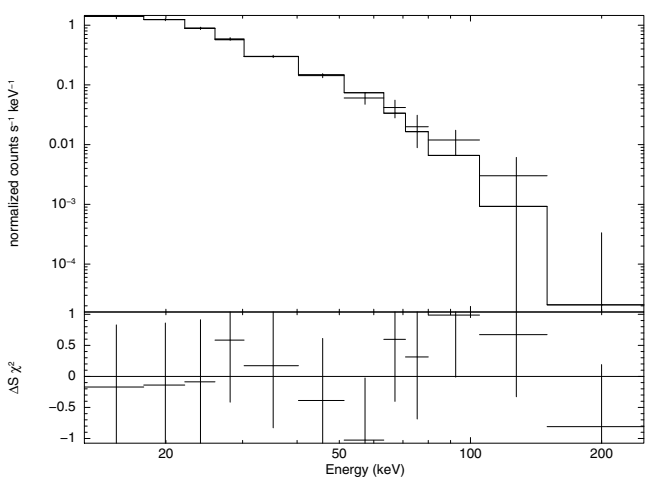

Fig. 5. The average spectrum of $4 \mathrm{U} \quad 2206+54$ from the INTEGRAL/IBIS observations during the second flare of the X-ray burst. The spectrum was fitted by a thermal bremsstrahlung model with $k T \sim 23.2 \mathrm{keV}$.

the first one. The first flare had a temporal profile of fast rise and exponential like decay, while the second one had a modulation period of $5550 \mathrm{~s}$ which is the pulsation period of the neutron star. (2) The flare had a hard spectrum. The first flare was only seen above $5 \mathrm{keV}$ from RXTE/ASM data; for the second flare, the $18-250 \mathrm{keV}$ spectrum was fitted by a thermal bremsstrahlung model with $k T \sim 23.4 \mathrm{keV}$ or a broken power-law model with the photon indexes $\Gamma_{1} \sim 2.3$ and $\Gamma_{2} \sim 3.3$, and a break energy of $E_{b} \sim 31 \mathrm{keV}$. No cyclotron absorption line during the outburst was detected. The long-duration flare has a mean X-ray luminosity of $\sim 1.3 \times 10^{36} \mathrm{erg} \mathrm{s}^{-1}$ and a total released energy higher than $10^{41}$ erg in the range of $20-150 \mathrm{keV}$.

These sudden strong X-ray flares have been detected in some other high-mass X-ray binaries (e.g., GX 301-2, see Haberl 1991; Leahy 1991; Koh et al. 1997; XTE J0052-723 Laycock et al. 2003). In these systems, the X-ray flares always occur before periastron passages of the neutron star. Therefore, the flares show the recurrence of orbital periodicity and have the following characteristics: (1) duration of $\sim 0.1$ orbital phase, roughly several days (Leahy 2002; Laycock et al. 2003); (2) strong photoelectric absorption, which makes the flares events absent at low energies ( $<5 \mathrm{keV}$, see Leahy 2002); (3) the spectrum can be described by a cut-off power-law model (Laycock et al. 2003). Recently, the X-ray outburst of a Be/X-ray pulsar A $0535+26$ was detected with a double-peak feature (Caballero et al. 2010). This outburst also showed the orbital modulations with a duration of more than ten days (orbital period $\sim 111$ days, Finger et al. 2006). In addition, the cyclotron absorption features were detected during the outburst, suggesting that the transient circumstellar-disk accretion near the periastron powered the outburst of A 0535+26 (Caballero et al. 2010).

Since these X-ray flares occurred with orbital periodicity, we can check this possible modulation in the X-ray light curve of $4 \mathrm{U} 2206+54$. If we assume the detected X-ray flare occurred around the periastron passage of the neutron star, then the X-ray flares would recur with the recurrence time of $\sim 19$ day. In Table 1, we presented the hard X-ray light curves (20-40 keV) of $4 \mathrm{U} 2206+54$ observed by IBIS-ISGRI covering two orbital periods, but only one flare was detected and no orbital modulation was found. Then the X-ray flare in 4U 2206+54 was different from the outbursts near the periastrons in other sources because the detected hard X-ray flare would not be repeated just because of the orbital modulation. Thus the hard X-ray flare in 4U 2206+54 may have other origins.

Recently, some soft gamma-ray time-structured bursts lasting from several hours to about 1 day have been detected by 
A\&A 520, A22 (2010)

Table 1. INTEGRAL/IBIS observations of the field around 4U 2206+54 from 2005 Dec. to 2006 Jan.

\begin{tabular}{lcccl}
\hline \hline Rev. Num. & Date & On-source time $(\mathrm{ks})$ & Mean count rate s $^{-1}$ & Detection level \\
\hline 384 & 2005 Dec. 05-07 & 118 & $2.86 \pm 0.24$ & $15 \sigma$ \\
385 & 2005 Dec. 08-10 & 132 & $2.01 \pm 0.20$ & $11 \sigma$ \\
386 & 2005 Dec. 11-13 & 121 & $1.66 \pm 0.19$ & $10 \sigma$ \\
387 & 2005 Dec. 14-16 & 98 & $11.9 \pm 0.25$ & $45 \sigma$ \\
388 & 2005 Dec. 17-19 & 103 & $2.38 \pm 0.21$ & $14 \sigma$ \\
389 & 2005 Dec. 20-22 & 110 & $1.38 \pm 0.30$ & $6 \sigma$ \\
390 & 2005 Dec. 23-25 & 102 & $0.66 \pm 0.25$ & $4.9 \sigma$ \\
391 & 2005 Dec. 26-28 & 113 & $1.78 \pm 0.21$ & $9 \sigma$ \\
392 & 2005 Dec. 29-31 & 110 & $2.58 \pm 0.22$ & $14 \sigma$ \\
393 & 2006 Jan. 01-03 & 118 & $0.77 \pm 0.21$ & $5.5 \sigma$ \\
394 & 2006 Jan. 04-06 & 115 & $1.68 \pm 0.20$ & $10 \sigma$ \\
395 & 2006 Jan. 07-09 & 124 & $1.61 \pm 0.21$ & $9 \sigma$ \\
\hline
\end{tabular}

INTEGRAL/IBIS observations in some supergiant high-mass $\mathrm{X}$-ray binaries, which were called supergiant fast X-ray transients (SFXTs, see Sidoli et al. 2005; Negueruela et al. 2006; Sguera et al. 2006, 2008). The SFXTs also belong to the windfed systems. The physical origin of the fast outbursts displayed in SFXTs is still unknown. It has been suggested that the presence of dense clumps in the wind of OB supergiant companions produces the accretion outbursts in SFXTs (in't Zand 2005; Walter \& Zurita Heras 2007). It is believed that SFXTs should contain sporadically accreting neutron stars, and they would be similar to the system of $4 \mathrm{U} 2206+54$. Some of the SFXTs have been found to contain a neutron star with a pulsation period from ten to a few hundred seconds (Sguera et al. 2007; Karasev et al. 2008), and an orbital period from several days to 30 days (Bird et al. 2009; Jain et al. 2009). 4U 2206+54 has a similar orbital period but a much slower pulsation period. The detected flare in $4 \mathrm{U} 2206+54$ also has a peak X-ray luminosity similar to those of SFXTs. Then the reported outbursts with X-ray luminosity higher than $10^{36} \mathrm{erg} \mathrm{s}^{-1}$ could just be a bright flare in $4 \mathrm{U} 2206+54$ caused by a suddenly enhanced wind accretion induced by an unknown process, which might be similar to the theoretical mechanism proposed in SFXTs. It is possible that the accretion materials as clumps or conglomeration from stellar winds hit the polar cap region of the highly magnetic neutron star to produce the hard X-ray outburst in 4U 2206+54.

The different effects on accretion by the wind of a main sequence star in 4U 2206+54 and supergiant winds in SFXTs still exist. More detailed study is clearly needed. It should be noted that a small part of the unidentified sources show the fast X-ray bursts and are candidate SFXTs though the optical counterpart has not yet been identified as the early-type supergiant star (see Negueruela et al. 2006; Sguera et al. 2006). Moreover, many early-type stars are characterized by highly structured and variable massive winds (Prinja et al. 2005). It is expected that this hard X-ray flare in 4U 2206+54 could be a missing link between these systems, so it may help us understand the nature of SFXTs and the flare of $4 \mathrm{U} 2206+54$.
Acknowledgements. The author is grateful to the referee for fruitful comments and Prof. C.K. Chou for useful discussions. This work was supported by the National Natural Science Foundation of China under grants 10803009 and 10833003 .

\section{References}

Bird, A. J., Bazzano, A., Hill, A. B., et al. 2009, MNRAS, 393, L11

Blay, P., Ribó, M., Negueruela, I., et al. 2005, A\&A, 438, 963

Blay, P., Negueruela, I., Reig, P., et al. 2006, A\&A, 466, 1095

Caballero, I., et al. 2010 [arXiv: 1003.2969]

Corbet, R. H. D., \& Peele, A. G. 2001, ApJ, 562, 936

Corbet, R. H. D., Markwardt, C. B., \& Tueller, J. 2007, ApJ, 655, 458

Finger, M. H., Camero-Arranz, A., Kretschmar, P., Wilson, C., \& Patel, S. 2006, BAAS, 38, 359

Finger, M. H., Ikhsanov, N. R., Wilson-Hodge, C. A., \& Patel, S. K. 2010, ApJ, 709, 1249

Giacconi, R., Murray, S., Gursky, H., et al. 1972, ApJ, 178, 281

Goldwurm, A., David, P., Foschini, L., et al. 2003, A\&A, 411, L223, 490, L161 Haberl, F. 1991, ApJ, 376, 245

In't Zand, J. J. M. 2005, A\&A, 441, L1

Jain, C., Paul, B., \& Dutta, A. 2009, MNRAS, 397, L11

Karasev, D.I., Tsygankov, S. S., \& Lutovinov, A. A. 2008, MNRAS, 386, L10

Koh, D. T., Bildsten, L., Chakrabarty, D., et al. 1997, ApJ, 479, 933

Laycock, S., Corbet, R. H. D., Coe, M. J., et al. 2003, MNRAS, 339, 435

Leahy, D. A. 1991, MNRAS, 250, 310

Leahy, D. A. 2002, A\&A, 391, 219

Lebrun, F., Leray, J. P., Lavocat, P., et al. 2003, A\&A, 411, L141

Masetti, N., Dal Fiume, D., Amati, L., et al. 2004, A\&A, 423, 311

Negueruela, I., \& Reig, P. 2001, A\&A, 371, 1056

Negueruela, I., Smith, D. M., Reig, P., Chaty, S., \& Torrejón, J. M. 2006, ESA SP, 604, 165

Prinja, R. K., Massa, D., \& Searle, S. C. 2005, A\&A, 430, L41

Reig, P., Torrejón, J. M., Negueruela, I., et al. 2009, A\&A, 494, 1073

Ribó, M., Negueruela, I., Blay, P., Torrejón, J. M., \& Reig, P. 2006, A\&A, 449, 687

Sguera, V., Bazzano, A., Bird, A. J., et al. 2006, ApJ, 646, 452

Sguera, V., Hill, A. B., Bird, A. J., et al. 2007, A\&A, 467, 249

Sguera, V., Bassani, L., Landi, R., et al. 2008, A\&A, 487, 619

Sidoli, L., Vercellone, S., Mereghetti, S., \& Tavani, M. 2005, A\&A, 429, L47

Torrejón, J. M., Kreykenbohm, I., Orr, A., Titarchuk, L., \& Negueruela, I. 2004, A\&A, 423, 301

Walter, R., \& Zurita Heras, J. A. 2007, A\&A, 476, 335

Wang, W. 2009, MNRAS, 398, 1428 